\title{
Manipulação de filtros ecológicos para aumentar a cobertura vegetal nativa em jazida tratada com lodo de esgoto no Bioma Cerrado
}

\author{
Management of ecological filters for increasing the native vegetation cover in a \\ mine treated with sewage sludge in the Cerrado Biome
}

\author{
Alexander Paulo do Carmo Balduíno ${ }^{\mathrm{I}}$, Rodrigo StudartCorrêa ${ }^{\mathrm{II}}$, \\ Cássia Beatriz Rodrigues Munhoz ${ }^{\mathrm{III}}$, Jair Eustáquio Quintino de Faria Júnior ${ }^{\mathrm{IV}}$, \\ João Bernardo de Azevedo Bringelv, Letícia dos Santos Barros ${ }^{\mathrm{VI}}$, \\ Pietro Matheus Pereira Santos ${ }^{\mathrm{VI}}$
}

\begin{abstract}
Resumo
Dominância de espécies exóticas invasoras e compactação de substratos minerados são filtros ecológicos que geralmente restringem o estabelecimento e o crescimento de espécies nativas de plantas em jazidas mineradas. O entendimento dos mecanismos de atuação e a eliminação desses filtros são de grande importância para a restauração ecológica de ecossistemas. Visando melhorar a condição ecológica de uma jazida tratada com lodo de esgoto dominada por Urochloa brizantha (Braquiarão) e com a superfície compactada, dois tratamentos foram aplicados em parcelas experimentais para se analisarem os efeitos da atenuação desses filtros sobre o recrutamento de plantas: uso de herbicida para redução da dominância de U. brizantha e escarificação mecanizada da superfície compactada, aplicados individualmente e combinados. A atenuação da dominância de $U$. brizanthae a descompactação da superfície minerada fomentaram o recrutamento de 29 espécies de plantas, $66 \%$ das quais descritas como espécies exóticas e invasoras do Cerrado. Os tratamentos aplicados individualmente ou combinados resultaram na formação de biocenoses mais ricas em espécies e mais biodiversas em comparação às parcelas usadas como controle. A cobertura de $U$. brizantha se recuperou até o final do período chuvoso nas parcelas que receberam os dois tratamentos, mas o recrutamento de espécies que permaneceram sobre a cobertura de U. brizantha melhorou a condição ecológica nas parcelas experimentais.
\end{abstract}

Palavras-chave: Glifosato; Compactação do solo; Restauração ecológica; Urochloa brizantha

\footnotetext{
Engenheiro Florestal, Dr., Analista Ambiental do Instituto Brasileiro do Meio Ambiente e dos Recursos Naturais Renováveis (IBAMA), SCEN Trecho 2, Edifício Sede, L4 Norte, CEP 70818-900, Brasília (DF) Brasil. ibama.alexander@gmail.com (ORCID: 0000-0003-4285-2685)

Engenheiro Agrônomo, Ph.D., Professor do Programa de Pós-Graduação em Ciências Ambientais, PPGCA/FUP, Universidade de Brasília, Caixa Postal 730, CEP 71510-970, Brasília (DF), Brasil. rscorrea@unb.br (ORCID: 0000-0002-9422-2629)

III Bióloga, Drª ., Professora do Departamento de Botânica, Instituto de Biologias, Universidade de Brasília, Asa Norte, Campus Darcy Ribeiro, CEP 70910-900, Brasília (DF), Brasil. cbrmunhoz@gmail.com (ORCID: 0000-0002-7990-6715)

IV Biólogo, Dr., Diretor de Gestão Integrada da Biodiversidade e Conscientização Pública, Superintendência Técnico-Científica, Jardim Botânico de Brasília, Área Especial SMDB Estação Ecológica Jardim Botânico de Brasília, CEP 71680-001, Brasília (DF), Brasil. jairfariajr@gmail.com (ORCID: 0000-0001-7875-6797)

Biólogo, Dr., Embrapa Recursos Genéticos e Biotecnologia (Bolsista DTI), Parque Estação Biológica, PqEB, Av. W5 Norte (final), Caixa Postal 02372, CEP 70770-917, Brasília (DF), Brasil. jbbringel@gmail.com (ORCID: 0000-0001-8423-2930)

vi Engenheira Florestal, Departamento de Engenharia Florestal, Universidade de Brasília, Posto Ecológico, Lado Oposto ao, Asa Norte, CEP 70297400, Brasília (DF), Brasil. leticiasbarros@outlook.com (ORCID: 0000-0003-1133-3399) / pietromatheus54@gmail.com (ORCID: 0000-0002-4525-1984)
} 


\begin{abstract}
The dominance of invasive species and the compaction of mine surfaces are ecological filters that usually restrict the establishment and growth of native plant species. The understanding of ecological filter mechanisms aiming to overcome their effects on the ecological succession is crucial for the ecological restoration of ecosystems. In order to evaluate the effects of reducing the intensity of the surface compaction and the grass dominance on the plant recruitment, we set up 24 experimental plots at six repetitions in a mine dominated by Urochloa brizantha. Treatments consisted in control (T1), herbicide application on $U$. brizantha (T2), mechanized plowing to ameliorate surface compaction (T3), and the combination of T2 and T3 (T4). Results showed that the chemical attenuation of U. brizantha dominance and surface decompaction allowed the recruitment of 29 plant species, $66 \%$ of which are invasive species, exotic to the Cerrado biome. However, the treatments T2, T3, and T4 resulted in the formation of richer and more diverse plant communities in comparison to the plots used as control (T1). U. brizantha cover redeveloped after both surface unpacking and herbicide application, but the plant species that recruited on $U$. brizantha cover in the meantime improved the ecological condition in experimental plots.
\end{abstract}

Keywords: Glyphosate; Soil compaction; Ecological restoration; Urochloa brizantha

\title{
Introdução
}

Competição com espécies gramíneas (Poaceae) invasoras e compactação de substratos são filtros ecológicos que retardam, dificultam ou impedem o estabelecimento e o desenvolvimento de plantas de espécies nativas em jazidas mineradas (HALASSY et al., 2016; SOLLENBERGER et al., 2016). Mensurar os efeitos da compactação de substratos minerados e da competição com gramíneas sobre espécies nativas e biocenoses é de grande importância para o desenvolvimento de técnicas de restauração de ecossistemas naturais (BUISSON et al., 2018).

Espécies de gramíneas invasoras possuem via metabólica $\mathrm{C}_{4}$, alta capacidade fotossintética, elevada capacidade de alocação de biomassa e nutrientes (LANNES et al., 2015), produzem substâncias alelopáticas e elevada quantidade de sementes com elevada e longa viabilidade (CASTILLIONI, 2015; CARAMASCHI etal., 2016). Essas características conferem a essas espécies a capacidade de excluírem espécies nativas (MARTINS et al., 2011; CARAMASCHI et al., 2016; PILON; BUISSON; DURIGAN, 2017) e alterarem o processo de sucessão ecológica em ambientes em processo de restauração (THOMAS, 2017; BUISSON et al., 2018).

A aplicação de herbicida figura entre os principais métodos utilizados para o controle de gramíneas exóticas invasoras. O uso de herbicida em áreas sob restauração é considerada prática eficiente para inibir a dominância dessas espécies e estimular o recrutamento de outras espécies vegetais (CAVA et al., 2016). Entre as formulações existentes no mercado, $\mathrm{N}$-fosfonometil glicina (Glifosato) é um herbicida sistêmico de amplo espectro, dessecante e de baixa toxicidade para animais (RODRIGUES, 2016). Trata-se de um dos herbicidas mais estudados no mundo e possui uma das maiores bases de dados entre os pesticidas (GOMES et al., 2014). O Glifosato tem sido utilizado em projetos de restauração ecológica com bons resultados sobre o controle de espécies vegetais invasoras (CAVA et al., 2016; MONTOANI; DIAS; TOREZAN, 2016). Entretanto, esse herbicida pode provocar alterações em fatores abióticos (luz, temperatura e disponibilidade de água) e bióticos (predação e interações facilitação/competição) que interagem com a germinação, o crescimento e a sobrevivência de plantas (GOMES et al., 2014). Portanto, seu uso para controlar espécies vegetais invasoras deve ser analisado em cada caso específico (MONTOANI; DIAS; TOREZAN, 2016).

Substratos minerados são compactados e a maioria dos estudos tem avaliado os efeitos da compactação sobre as propriedades físico-hídricas de solos (CORREAA; BENTO, 2010). Poucos estudos avaliaram os efeitos da compactação de substratos minerados sobre o recrutamento de plantas. Os primeiros trabalhos desenvolvidos na região do Cerrado concluíram que qualquer tentativa de revegetação de substratos minerados requer escarificação seguida de adição de matéria orgânica (BORGES et al., 2009; CORRÊA et al., 2018).

Em face do exposto, este trabalho testou as hipóteses de que o controle químico de Urochloa brizantha (Braquiarão) e a descompactação do substrato revegetado, onde essa espécie domina, reduzirão a intensidade de atuação dos filtros competição e compactação e estimularão o recrutamento de outras espécies vegetais na jazida revegetada. 


\section{Materiais e métodos}

\section{Caracterização da área de estudo}

O estudo foi conduzido em uma jazida de cascalho localizada na porção sudoeste do Distrito Federal, à margem da Rodovia BR-060, sentido Brasília-Goiânia (Figura 1). A jazida fora explorada entre 2000 e 2001, restando uma cava de 67 ha e $4 \mathrm{~m}$ de profundidade. Em 2002, 250 $\mathrm{Mg} \mathrm{ha}^{-1}$ de lodo de esgoto doméstico proveniente de tratamento terciário e com $12 \%$ de sólidos foram mecanicamente incorporados à camada superficial $(0-15 \mathrm{~cm})$ do substrato exposto pela mineração. Uma densa cobertura herbácea se estabeleceu na área nos anos seguintes. Em 2005, capim braquiarão (Urochloa brizantha) foi semeado $\left(7 \mathrm{~kg} \mathrm{ha}^{-1}\right)$ no substrato tratado com lodo de esgoto, sendo a área deste então utilizada como pasto para cerca de trinta bovinos $(0,5$ cabeça/ hectare). Na paisagem minerada foram deixados blocos de solo original (damas), classificado como Cambissolo háplico (EMBRAPA, 2013), sob fragmentos de Cerrado sensu stricto. Um fragmento de 4,24 ha de Cerrado sensu stricto foi poupado durante a atividade de mineração (Figura 1). O

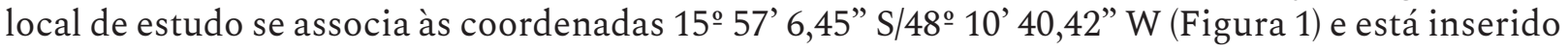
na região da Chapada do Divisor Descoberto-Alagado (COMPANHIA DE PLANEJAMENTO DO DISTRITO FEDERAL, 2018). O clima local é classificado como Tropical de Savana (Aw Köppen-Geiger), com pluviosidade média anual de $1.560 \mathrm{~mm}$ e temperaturas médias oscilando entre $18.5^{\circ} \mathrm{C}$ e $21.8^{\circ} \mathrm{C}$ (CLIMATE-DATA.ORG, 2018).

\section{Figura 1 - Localização da jazida estudada e das parcelas experimentais}

Figure 1 - Location of the studied mined deposit and the experimental plots

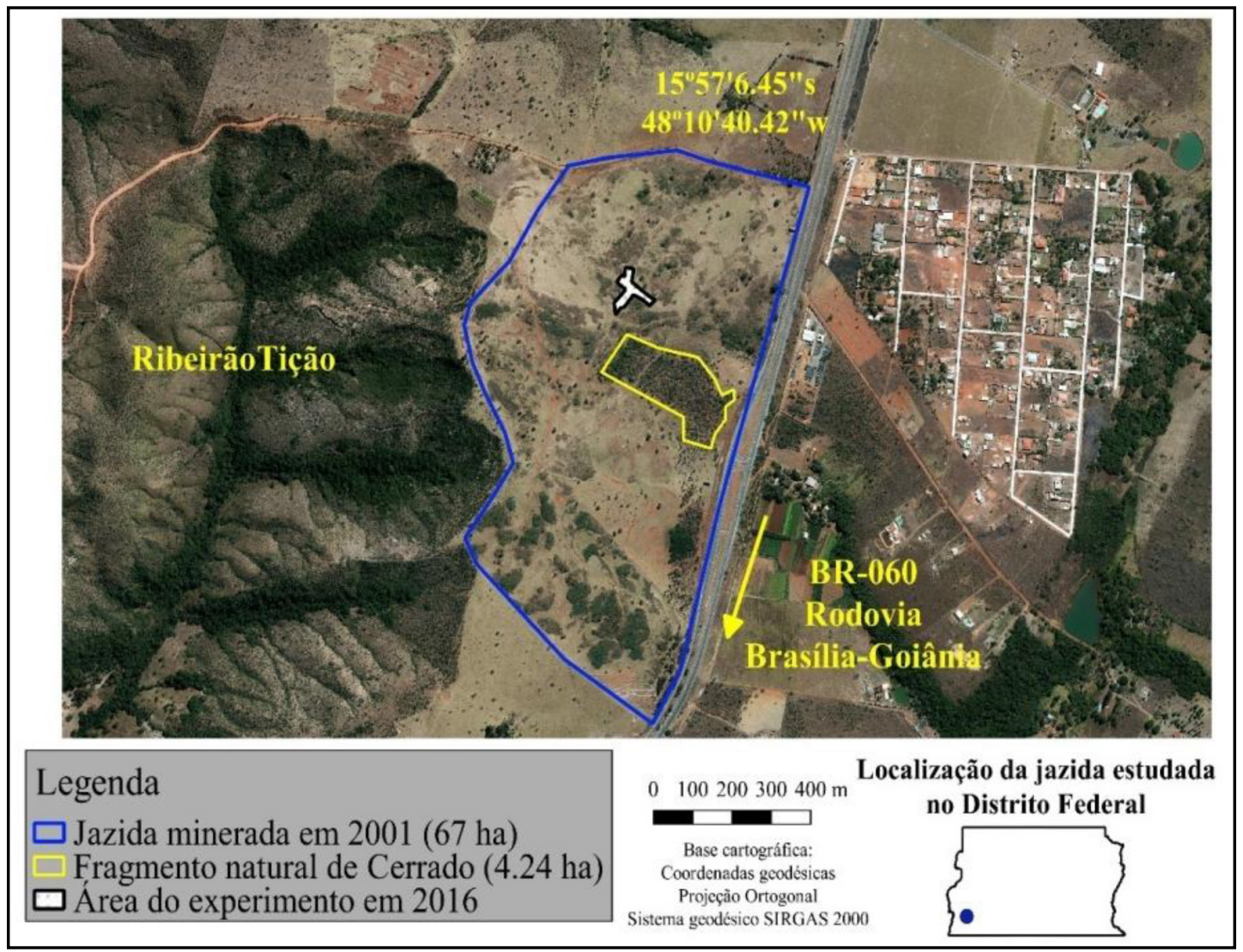

Fonte: Autores (2019) 


\section{Delineamento experimental}

Para testar as hipóteses formuladas neste trabalho, foram estabelecidas 24 parcelas experimentais de $100 \mathrm{~m}^{2}$ cada uma $(10 \times 10 \mathrm{~m})$, totalizando 0,24 ha de área amostrada. As parcelas foram distribuídas em seis blocos $\left(\mathrm{BL}_{1}\right.$ a $\left.\mathrm{BL}_{6}\right)$ de $400 \mathrm{~m}^{2}$ cada um $(20 \times 20 \mathrm{~m})$, em sítios que apresentavam 100\% de cobertura vegetal formada por Urochloa brizantha (Braquiarão) (Figura 2). Todos os blocos foram cercados com arames montados em estacas de madeira para evitar a interferência de animais domésticos.

Três blocos $\left(\mathrm{BL}_{1}\right.$ a $\left.\mathrm{BL}_{3}\right)$, totalizando doze parcelas, foram implantados sobre a superfície compactada da jazida revegetada e três blocos $\left(\mathrm{BL}_{4}\right.$ a $\left.\mathrm{BL}_{6}\right)$, totalizando outras doze parcelas, foram implantados em uma faixa mecanicamente escarificada para a implantação deste experimento. Uma grade de discos (14 x 28”) acoplada a um trator de pneus (New Holland 7630) foi utilizada no trabalho de escarificação da superfície revegetada. A superfície compactada apresentava 1,43 $\pm 0,09 \mathrm{Mg} \mathrm{m}^{-3}$ de densidade aparente e a escarificação reduziu esse valor para 1,12 $\pm 0,07 \mathrm{Mg} \mathrm{m}^{-3}$. Em seis parcelas com a superfície escarificada e em seis parcelas não escarificadas aplicaram-se $200 \mathrm{ml} \mathrm{m}^{-2}$ de Glifosato a 5\% de concentração aos 30 e aos 60 dias após a implantação das parcelas. Seis parcelas com a superfície escarificada e seis parceladas não escarificadas permaneceram isentas da aplicação de herbicida.

Dessa forma, o delineamento experimental consistiu de seis parcelas utilizadas como testemunha $\left(T_{1}\right)$, seis parcelas que receberam herbicida $\left(T_{2}\right)$, seis parcelas em que a superfície fora escarificada $\left(\mathrm{T}_{3}\right)$ e seis em que se combinou a superfície escarificada com a aplicação de herbicida $\left(\mathrm{T}_{4}\right)$.

Figura 2 - Delineamento experimental. Testemunha $\left(T_{1}\right)$ e tratamentos $\left(T_{2}, T_{3}\right.$ e $\left.T_{4}\right)$ foram alocados nos blocos BL1 a BL6

Figure 2 - Experimental design. Control (T1) and treatments (T2, T3 and T4) were allocated in the blocks BL1 to BL6

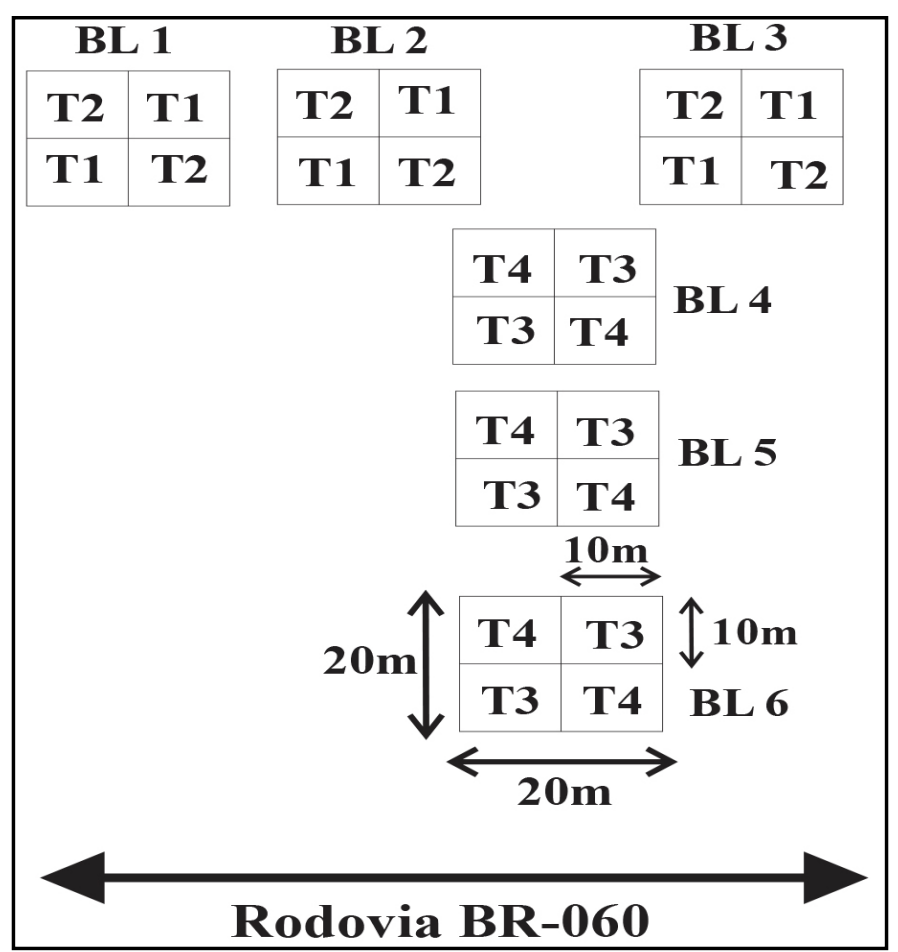

Fonte: Autores (2019) 


\section{Fitossociologia das comunidades vegetais}

Transcorridos 365 dias da instalação do experimento, foram efetuados levantamentos fitossociológicos nas parcelas experimentais, quando as plantas recrutadas foram amostradas pelo Método de Interseção em Linha (MUNHOZ; ARAUJO, 2011). Para cada parcela de 100 $\mathrm{m}^{2}(10 \times 10 \mathrm{~m})$ foram traçadas cinco linhas com 9,2 $\mathrm{m}$ de comprimento (foram descontados $0,80 \mathrm{~m}$ relativos ao efeito de borda de parcelas vizinhas) separadas a equidistâncias de 1,5 m. Plantas não identificadas no local foram coletadas, prensadas e identificadas no Herbário da Universidade de Brasília. Nomes científicos foram atualizados conforme a Flora do Brasil 2020 (JARDIM BOTÂNICO DO RIO DE JANEIRO, 2019). Após compilação da flora amostrada, as espécies foram classificadas segundo origem (nativa ou exótica e invasora ao Cerrado) e hábito (erva, trepadeira, subarbusto, arbusto ou árvore). Para avaliar o recrutamento como resposta aos tratamentos, foram estimados em cada parcela experimental e no conjunto de parcelas $\mathrm{T}_{1}, \mathrm{~T}_{2}, \mathrm{~T}_{3}$ e $\mathrm{T}_{4}$ a cobertura absoluta de espécies $\left(\mathrm{CA}_{\mathrm{i}}\right)$, a frequência absoluta de espécies $\left(\mathrm{FA}_{\mathrm{i}}\right)$, a cobertura absoluta de Urochloa brizantha (CUB), a cobertura absoluta de espécies nativas (CN), a diversidade de Shannon geral $\left(\mathrm{H}^{\prime}\right)$ e a de espécies nativas $\left(\mathrm{H}^{\prime} \mathrm{N}\right)$, e a riqueza geral ( $\left.\mathrm{S}^{\prime}\right)$ e a de espécies nativas $\left(S^{\prime} N\right)$. O processamento de dados e a estimativa dos parâmetros fitossociológicos foram realizados no programa Microsoft Excel 2016.

\section{Análise estatística}

A similaridade florística entre parcelas testemunhas $\left(T_{1}\right)$ e parcelas que receberam os tratamentos $\left(\mathrm{T}_{2}, \mathrm{~T}_{3}\right.$ e $\left.\mathrm{T}_{4}\right)$ foi analisada pela técnica Non metric Multidimensional Scaling - NMDS (FELFILI et al., 2011). Para verificar o efeito da aplicação de herbicida $\left(\mathrm{T}_{2}\right)$, da escarificação da superfície revegetada $\left(\mathrm{T}_{3}\right)$ e da interação entre o herbicida e a escarificação $\left(\mathrm{T}_{4}\right)$ sobre o recrutamento de plantas e sobre os parâmetros calculados, foi utilizado o Modelo Linear Generalizado associado à Análise de Variância (ANOVA). Foram calculados intervalos de confiança simultâneos e realizadas comparações pareadas pelo teste de Tukey $(\mathbb{Z}=95 \%)$ para as médias dos fatores combinados (testemunha + tratamentos). A normalidade de dados foi avaliada pelos testes de Bartlett e de Levene $(\alpha=95 \%)$, sendo efetuadas transformações por raiz quadrada em dados não normalizados. As análises estatísticas foram executadas nos softwares $\mathrm{R}$ versão 3.3.1 e MINITAB 18.

\section{Resultados e discussão}

As parcelas da área de 0,24 ha que receberam controle químico de Urochloa brizantha (Braquiarão) e/ou a escarificação mecanizada da superfície revegetada $\left(\mathrm{T}_{2}\right.$ a $\left.\mathrm{T}_{4}\right)$ foram colonizadas por 29 espécies de plantas pertencentes a 14 famílias botânicas (Tabela 1). Das espécies que recrutaram no local, $76 \%$ são ervas ou subarbustos e $66 \%$ são descritas como exóticas e invasoras ao Cerrado (Tabela 1). Passados 365 dias da implantação do experimento, U. brizantha regenerouse e apresentou forte dominância de cobertura e frequência em relação às demais espécies amostradas, independentemente do tratamento aplicado (Tabela 1). Essa espécie esteve presente em todas as 24 parcelas amostradas $\left(\mathrm{FA}_{\mathrm{i}}=100 \%\right)$. Outras espécies que ocuparam posições de destaque na cobertura das parcelas foram Andropogon gayanus $\left(\mathrm{T}_{1}\right)$, Ricinus communis $\left(\mathrm{T}_{2}, \mathrm{~T}_{3}\right.$ e $\left.\mathrm{T}_{4}\right)$, Mesosphaerum pectinatum $\left(\mathrm{T}_{2}, \mathrm{~T}_{3}\right.$ e $\left.\mathrm{T}_{4}\right)$, Triumfetta rhomboidea $\left(\mathrm{T}_{3}\right.$ e $\left.\mathrm{T}_{4}\right)$ e Sida glaziovii $\left(\mathrm{T}_{4}\right)$, sendo esta última espécie nativa ao Cerrado (Tabela 1). 
Tabela 1 - Fitossociologia das biocenoses vegetais que colonizaram as 24 parcelas experimentais

Table 1 - Phytosociology of plant biocenoses that colonized the 24 experimental plots

\begin{tabular}{|c|c|c|c|c|c|}
\hline \multicolumn{6}{|c|}{$\mathrm{T}_{1}($ Testemunha $)$} \\
\hline Espécie & Família & Origem* & Hábito* & $\begin{array}{l}\mathbf{C A}_{\mathrm{i}} \\
(\mathbf{m})\end{array}$ & $\begin{array}{l}\text { FA }_{i} \\
(\%)\end{array}$ \\
\hline $\begin{array}{l}\text { Urochloa brizantha (Hochst. ex A. Rich.) R.D. } \\
\text { Webster }\end{array}$ & Poaceae & EI & erva & 266,12 & 100,00 \\
\hline Andropogon gayanus Kunth & Poaceae & EI & erva & 8,65 & 20,00 \\
\hline Sida glaziovii K. Schum. & Malvaceae & $\mathrm{N}$ & subarbusto & 0,40 & 13,33 \\
\hline Mesosphaerum pectinatum (L.) Kuntze & Lamiaceae & EI & subarbusto & 0,27 & 3,33 \\
\hline Triumfetta rhomboidea Jacq. & Malvaceae & EI & subarbusto & 0,27 & 6,67 \\
\hline Lepidaploa aurea (Mart. ex DC.) H. Rob. & Asteraceae & $\mathrm{N}$ & arbusto & 0,15 & 3,33 \\
\hline Melinis repens (Willd.) Zizka & Poaceae & EI & erva & 0,08 & 3,33 \\
\hline Stylosanthes cf scabra Vogel & Fabaceae & $\mathrm{N}$ & erva & 0,06 & 3,33 \\
\hline Totais & & & & 276,00 & 153,33 \\
\hline \multicolumn{6}{|c|}{$\mathbf{T}_{2}$ (aplicação de herbicida) } \\
\hline Espécie & Família & Origem & Hábito & $\begin{array}{c}\text { CAi } \\
(\mathbf{m})\end{array}$ & $\begin{array}{c}\text { FAi } \\
(\%)\end{array}$ \\
\hline $\begin{array}{l}\text { Urochloa brizantha (Hochst. ex A. Rich.) R.D. } \\
\text { Webster }\end{array}$ & Poaceae & EI & erva & 220,85 & 100,00 \\
\hline Ricinus communis $\mathbf{L}$. & Euphorbiaceae & EI & subarbusto & 19,26 & 60,00 \\
\hline Mesosphaerum pectinatum (L.) Kuntze & Lamiaceae & EI & subarbusto & 10,16 & 33,33 \\
\hline Andropogon gayanus Kunth & Poaceae & EI & erva & 9,34 & 16,67 \\
\hline Sida glaziovii K. Schum. & Malvaceae & $\mathrm{N}$ & subarbusto & 3,17 & 46,67 \\
\hline Triumfetta rhomboidea Jacq. & Malvaceae & EI & subarbusto & 2,31 & 10,00 \\
\hline Solanum paniculatum $\mathrm{L}$. & Solanaceae & EI & arbusto & 2,12 & 13,33 \\
\hline Lepidaploa aurea (Mart. ex DC.) H.Rob. & Asteraceae & $\mathrm{N}$ & arbusto & 1,02 & 6,67 \\
\hline Sidastrum micranthum (A. St.-Hil.) Fryxell & Malvaceae & $\mathrm{N}$ & erva & 0,81 & 20,00 \\
\hline Sida acuta Burm.f. & Malvaceae & EI & subarbusto & 0,76 & 6,67 \\
\hline Momordica charantia L. & Cucurbitaceae & EI & $\begin{array}{l}\text { erva } \\
\text { trepadeira }\end{array}$ & 0,71 & 3,33 \\
\hline Verbena litoralis Kunth & Plantaginaceae & EI & erva & 0,65 & 3,33 \\
\hline $\begin{array}{l}\text { Chromolaena maximiliani (DC. ex Schrad.) R.M. } \\
\text { King \& H. Rob. }\end{array}$ & Asteraceae & $\mathrm{N}$ & arbusto & 0,45 & 6,67 \\
\hline Solanum falciforme Farruggia. & Solanaceae & $\mathrm{N}$ & árvore & 0,45 & 3,33 \\
\hline Sida rhombifolia $\mathrm{L}$. & Malvaceae & EI & subarbusto & 0,38 & 6,67 \\
\hline
\end{tabular}


Tabela 1 - Continuação ...

Table 1 - Continuation ...

\begin{tabular}{|c|c|c|c|c|c|}
\hline \multicolumn{6}{|c|}{$\mathbf{T}_{2}$ (aplicação de herbicida) } \\
\hline Espécie & Família & Origem & Hábito & $\begin{array}{l}\text { CAi } \\
(\mathbf{m})\end{array}$ & $\begin{array}{l}\text { FAi } \\
(\%)\end{array}$ \\
\hline Tridax procumbens $\mathrm{L}$. & Asteraceae & EI & erva & 0,34 & 3,33 \\
\hline Solanum viarum Dunal & Solanaceae & $\mathrm{N}$ & arbusto & 0,33 & 6,67 \\
\hline $\begin{array}{l}\text { Vernonanthura polyanthes (Spreng.) A.J. Vega \& M. } \\
\text { Dematt. }\end{array}$ & Asteraceae & $\mathrm{N}$ & arbusto & 0,30 & 6,67 \\
\hline Asclepias curassavica L. & Apocynaceae & EI & erva & 0,10 & 3,33 \\
\hline Totais & & & & 276,00 & 373,33 \\
\hline \multicolumn{6}{|c|}{$\mathbf{T}_{3}$ (escarificação mecânica do substrato) } \\
\hline Espécie & Família & Origem & Hábito & $\begin{array}{c}\text { CAi } \\
(\mathbf{m})\end{array}$ & $\begin{array}{l}\text { FAi } \\
(\%)\end{array}$ \\
\hline $\begin{array}{l}\text { Urochloa brizantha (Hochst. ex A. Rich.) R.D. } \\
\text { Webster }\end{array}$ & Poaceae & EI & erva & 228,99 & 100,00 \\
\hline Ricinus communis $\mathbf{L}$. & Euphorbiaceae & EI & subarbusto & 14,86 & 33,33 \\
\hline Mesosphaerum pectinatum (L.) Kuntze & Lamiaceae & EI & subarbusto & 8,49 & 63,33 \\
\hline Triumfetta rhomboidea Jacq. & Malvaceae & EI & subarbusto & 7,05 & 50,00 \\
\hline Stachytarpheta elatior Schrad. ex Schult. & Verbenaceae & $\mathrm{N}$ & subarbusto & 6,01 & 23,33 \\
\hline Sidastrum micranthum (A. St.-Hil.) Fryxell & Malvaceae & $\mathrm{N}$ & erva & 3,16 & 36,67 \\
\hline Sida glaziovii K. Schum. & Malvaceae & $\mathrm{N}$ & subarbusto & 1,70 & 43,33 \\
\hline Solanum viarum Dunal & Solanaceae & $\mathrm{N}$ & arbusto & 1,44 & 13,33 \\
\hline $\begin{array}{l}\text { Chromolaena maximiliani (DC. ex Schrad.) R.M. } \\
\text { King \& H. Rob. }\end{array}$ & Asteraceae & $\mathrm{N}$ & arbusto & 1,22 & 13,33 \\
\hline Melochia parvifolia Kunth & Malvaceae & EI & erva & 0,91 & 13,33 \\
\hline Sida rhombifolia $\mathbf{L}$. & Malvaceae & EI & subarbusto & 0,63 & 20,00 \\
\hline Cleome spinosa Jacq. & Cleomaceae & EI & subarbusto & 0,54 & 3,33 \\
\hline $\begin{array}{l}\text { Ageratum fastigiatum (Gardner) R.M. King \& H. } \\
\text { Rob. }\end{array}$ & Asteraceae & $\mathrm{N}$ & erva & 0,30 & 6,67 \\
\hline Euploca procumbens (Mill.) Diane \& Hilger & Heliotropiaceae & EI & erva & 0,20 & 3,33 \\
\hline Asclepias curassavica $\mathrm{L}$. & Apocynaceae & EI & erva & 0,15 & 3,33 \\
\hline Cynodon sp & Poaceae & - & erva & 0,11 & 3,33 \\
\hline Heliotropium indicum $\mathbf{L}$. & Boraginaceae & EI & erva & 0,10 & 3,33 \\
\hline Tagetes minuta $\mathrm{L}$. & Asteraceae & EI & erva & 0,10 & 3,33 \\
\hline $\begin{array}{l}\text { Vernonanthura polyanthes (Spreng.) A.J. Vega \& M. } \\
\text { Dematt. }\end{array}$ & Asteraceae & $\mathrm{N}$ & arbusto & 0,04 & 3,33 \\
\hline Total Geral & & & & 276,00 & 440,00 \\
\hline
\end{tabular}


Tabela 1 - Conclusão ...

Table 1 - Conclusion ...

\begin{tabular}{|c|c|c|c|c|c|}
\hline \multicolumn{6}{|c|}{$\mathrm{T}_{4}$ (aplicação de herbicida e escarificação mecânica do substrato) } \\
\hline Espécie & Família & Origem & Hábito & $\begin{array}{c}\text { CAi } \\
(\mathbf{m})\end{array}$ & $\begin{array}{c}\text { FAi } \\
(\%)\end{array}$ \\
\hline $\begin{array}{l}\text { Urochloa brizantha (Hochst. ex A. Rich.) R.D. } \\
\text { Webster }\end{array}$ & Poaceae & EI & erva & 215,35 & 100,00 \\
\hline Sida glaziovii K. Schum. & Malvaceae & $\mathrm{N}$ & subarbusto & 17,58 & 80,00 \\
\hline Triumfetta rhomboidea Jacq. & Malvaceae & EI & subarbusto & 12,68 & 66,67 \\
\hline Ricinus communis $\mathbf{L}$. & Euphorbiaceae & EI & subarbusto & 9,85 & 36,67 \\
\hline Mesosphaerum pectinatum (L.) Kuntze & Lamiaceae & EI & subarbusto & 9,06 & 86,67 \\
\hline Sidastrum micranthum (A. St.-Hil.) Fryxell & Malvaceae & $\mathrm{N}$ & erva & 4,91 & 70,00 \\
\hline Cleome spinosa Jacq. & Cleomaceae & EI & subarbusto & 1,89 & 6,67 \\
\hline Sida rhombifolia $\mathrm{L}$. & Malvaceae & EI & subarbusto & 1,27 & 33,33 \\
\hline $\begin{array}{l}\text { Chromolaena maximiliani (DC. ex Schrad.) R.M. } \\
\text { King \& H. Rob. }\end{array}$ & Asteraceae & $\mathrm{N}$ & arbusto & 0,60 & 6,67 \\
\hline Verbena litoralis Kunth & Plantaginaceae & EI & erva & 0,53 & 13,33 \\
\hline Melochia parvifolia Kunth & Malvaceae & EI & erva & 0,51 & 10,00 \\
\hline Sida acuta Burm.f. & Malvaceae & EI & subarbusto & 0,43 & 6,67 \\
\hline Lepidaploa aurea (Mart. ex DC.) H.Rob. & Asteraceae & $\mathrm{N}$ & arbusto & 0,35 & 3,33 \\
\hline Solanum falciforme Farruggia. & Solanaceae & $\mathrm{N}$ & árvore & 0,23 & 3,33 \\
\hline $\begin{array}{l}\text { Ageratum fastigiatum (Gardner) R.M. King \& H. } \\
\text { Rob. }\end{array}$ & Asteraceae & $\mathrm{N}$ & erva & 0,18 & 3,33 \\
\hline Solanum viarum Dunal & Solanaceae & $\mathrm{N}$ & arbusto & 0,15 & 3,33 \\
\hline Total Geral & & & & 276,00 & 536,67 \\
\hline
\end{tabular}

Fonte: Jardim Botânico do Rio de Janeiro (2019)

Em que: testemunha $=\mathrm{T} 1$ e tratamentos $=\mathrm{T}_{2}, \mathrm{~T}_{3}$ e $\mathrm{T}_{4} . \mathrm{EI}=$ Exótica e invasora ao Cerrado. $\mathrm{N}=$ Nativa ao Cerrado. $\mathrm{CA}_{\mathrm{i}}$ = Cobertura absoluta da espécie $\mathrm{i}$. $\mathrm{FA}_{\mathrm{i}}=$ Frequência absoluta da espécie $\mathrm{i}$.

Os valores de diversidade de Shannon e a riqueza de espécies foram mais elevados nas parcelas que receberam os tratamentos $\left(\mathrm{T}_{2}, \mathrm{~T}_{3}\right.$ e $\left.\mathrm{T}_{4}\right)$ em relação às parcelas usadas como testemunha $\left(\mathrm{T}_{1}\right)$ (Tabela 2). U. brizantha reduziu significativamente sua dominância na cobertura vegetal das parcelas que receberam controle químico e escarificação da superfície revegetada $\left(\mathrm{T}_{4}\right)$ em relação às parcelas que receberam os outros tratamentos $\left(\mathrm{T}_{1} \mathrm{a}_{3}\right)$. Da mesma forma, a cobertura vegetal formada por espécies nativas ao Cerrado foi significativamente maior em $\mathrm{T}_{4}$ do que nas parcelas que receberam os outros tratamentos $\left(T_{1}\right.$ a $\left.T_{3}\right)$ (Tabela 2).

A ordenação espacial dos dados, realizada pelo Método de Escalonamento Multidirecional Não Métrico - NMDS (Figura 3), mostrou elevada dissimilaridade florístico-estrutural entre as parcelas testemunhas $\left(\mathrm{T}_{1}\right)$ e as parcelas que receberam os tratamentos $\left(\mathrm{T}_{2}, \mathrm{~T}_{3}\right.$ e $\left.\mathrm{T}_{4}\right)$. As comunidades que recrutaram sobre as parcelas submetidas aos tratamentos mostram-se floristicamente e estruturalmente semelhantes entre si (Figura 3). 
Tabela 2 - Parâmetros fitossociológicos estimados nas parcelas testemunhas e nas parcelas que receberam os tratamentos

Table 2 - Estimated phytosociological parameters in the control plots and in the plots receiving the treatments

\begin{tabular}{lcccccc}
\hline Biocenose & $\mathbf{H}^{\prime}$ & H'N $^{\prime}$ & $\mathbf{S}$ & $\mathbf{S N}$ & $\mathbf{C U B}(\mathbf{m})$ & $\mathbf{C N}(\mathbf{m})$ \\
\hline $\mathbf{T}_{1}$ & 0,175 & 0,015 & 8 & 3 & 266,12 & 0,61 \\
$\mathbf{T}_{2}$ & 0,912 & 0,126 & 19 & 7 & 220,85 & 6,53 \\
$\mathbf{T}_{3}$ & 0,802 & 0,226 & 19 & 7 & 228,99 & 13,98 \\
$\mathbf{T}_{4}$ & 0,953 & 0,284 & 16 & 7 & 215,35 & 24 \\
\hline
\end{tabular}

Fonte: Autores (2019)

Em que: testemunha $=\mathrm{T} 1$ e tratamentos $=\mathrm{T}_{2}, \mathrm{~T}_{3}$ e $\mathrm{T}_{4} \mathrm{H}^{\prime}=$ diversidade de Shannon geral. $\mathrm{H}^{\prime} \mathrm{N}=$ diversidade de Shannon de espécies nativas ao Cerrado. $\mathrm{S}$ = riqueza geral. $\mathrm{SN}$ = riqueza de espécies nativas ao Cerrado. CUB = cobertura absoluta de Urochloa brizantha. $\mathrm{CN}$ = cobertura absoluta de espécies nativas ao Cerrado.

Os resultados da ANOVA associada ao Modelo Linear Generalizado (Tabela 3) mostraram que o herbicida alterou significativamente os valores da diversidade de Shannon geral (H'), diversidade de Shannon de espécies nativas $\left(\mathrm{H}^{\prime} \mathrm{N}\right)$ e riqueza de espécies nativas $(\mathrm{SN})$ em relação ao controle do experimento $\left(\mathrm{T}_{1}\right)$. $\mathrm{O}$ efeito da escarificação do substrato foi significativo para todos os parâmetros fitossociológicos analisados, exceto para a cobertura absoluta de Urochloa brizantha (CUB). A aplicação do herbicida combinada à escarificação da superfície compactada não foi eficiente para significativamente reduzir a cobertura absoluta (CUB) de Urochloa brizantha (Tabela 3). As comunidades vegetais nas parcelas submetidas aos tratamentos apresentaram valores médios da diversidade de Shannon geral ( $\left.\mathrm{H}^{\prime}\right)$ e de riqueza geral (S) significativamente maiores do que a parcelas usadas como testemunhas $\left(\mathrm{T}_{1}\right)$ (Figura 4). Não houve diferença significativa da diversidade de Shannon de espécies nativas $\left(\mathrm{H}^{\prime} \mathrm{N}\right)$ e da riqueza de espécies nativas (SN) entre as biocenoses das parcelas testemunhas $\left(\mathrm{T}_{1}\right)$ e aquelas recrutadas após a aplicação de herbicida $\left(\mathrm{T}_{2}\right)$. Todavia, as biocenoses em $\mathrm{T}_{1}$ diferenciaram-se significativamente daquelas sobre as parcelas submetidas aos tratamentos $\mathrm{T}_{3}$ e $\mathrm{T}_{4}$ (Figura 4). Os tratamentos aplicados $\left(\mathrm{T}_{2}, \mathrm{~T}_{3}\right.$ e $\mathrm{T}_{4}$ ) não surtiram efeito significativo sobre a cobertura absoluta de $U$. brizantha, que permaneceu similar àquela mensurada nas parcelas testemunhas $\left(\mathrm{T}_{1}\right)$ (Figura 4). Comunidades vegetais nas parcelas do tratamento $\mathrm{T}_{4}$ alcançaram o maior valor de cobertura absoluta de espécies nativas $(\mathrm{CN})$, que diferiu significativamente apenas das parcelas testemunhas $\left(\mathrm{T}_{1}\right)$ (Figura 4).

Filtros ecológicos moldam comunidades bióticas (HALASSY etal., 2016; SOLLENBERGER et al., 2016) e a manipulação dos filtros competição e compactação da superfície, neste trabalho, alterou o equilíbrio previamente existente na área de estudo e afetou a colonização de plantas na jazida revegetada. A aplicação de herbicida para reduzir a dominância do capim Urochloa brizantha e a escarificação do substrato aumentaram significativamente a riqueza e a diversidade de espécies de plantas espontaneamente recrutadas na área de estudo (Figura 4). A composição de espécies e os respectivos valores de cobertura absoluta variaram em função do tratamento dispensado às parcelas experimentais (Tabela 1; Figura 3).

Aumentar a diversidade de espécies nativas e reduzir a cobertura vegetal de espécies exóticas e invasoras são metas em áreas sob restauração ecológica (FLORY; CLAY, 2009; PILON; BUISSON; DURIGAN, 2017). Nesse sentido, o uso de herbicidas em áreas dominadas por espécies gramíneas (Poaceae) é considerado manejo adequado que visa a aumentar a contribuição de espécies nativas em comunidades sob restauração (CASTILLIONI, 2015; MONTOANI; DIAS; TOREZAN, 2016; THOMAS, 2017). Essa técnica, associada ao uso do fogo, foi capaz de praticamente eliminar a presença de capins invasores em ecossistemas savânicos e permitir o 
recrutamento de outras espécies (BUISSON et al., 2018). Similarmente, a aplicação de herbicida em uma pastagem induziu a regeneração natural em área de Cerrado (CAVA et al., 2016).

Figura 3 - Análise de ordenação por Escalonamento Multidirecional Não Métrico (NMDS) em função da composição florística e cobertura absoluta $(m)$. Parcelas experimentais $=T_{1} R_{1} a$ $\mathbf{T}_{4} \mathbf{R}_{6} \cdot \mathbf{T}_{1}=$ testemunha e $\mathbf{T}_{2}, \mathbf{T}_{3}$ e $\mathbf{T}_{4}=$ tratamentos. Repetições $=\mathbf{R}_{1}$ a $\mathbf{R}_{6}$. Valor de stress $=\mathbf{0 , 0 9 0}$

Figure 3 - Multidirectional Non-Metric Scheduling (NMDS) ordering as a function of floristic composition and absolute coverage $(\mathrm{m})$. Experimental plots $=\mathrm{T} 1 \mathrm{R} 1$ to T4R6. T1 = control and

$\mathrm{T} 2, \mathrm{~T} 3$ and $\mathrm{T} 4=$ treatments. Repetitions $=\mathrm{R} 1$ to $\mathrm{R} 6$. Stress value $=0.090$

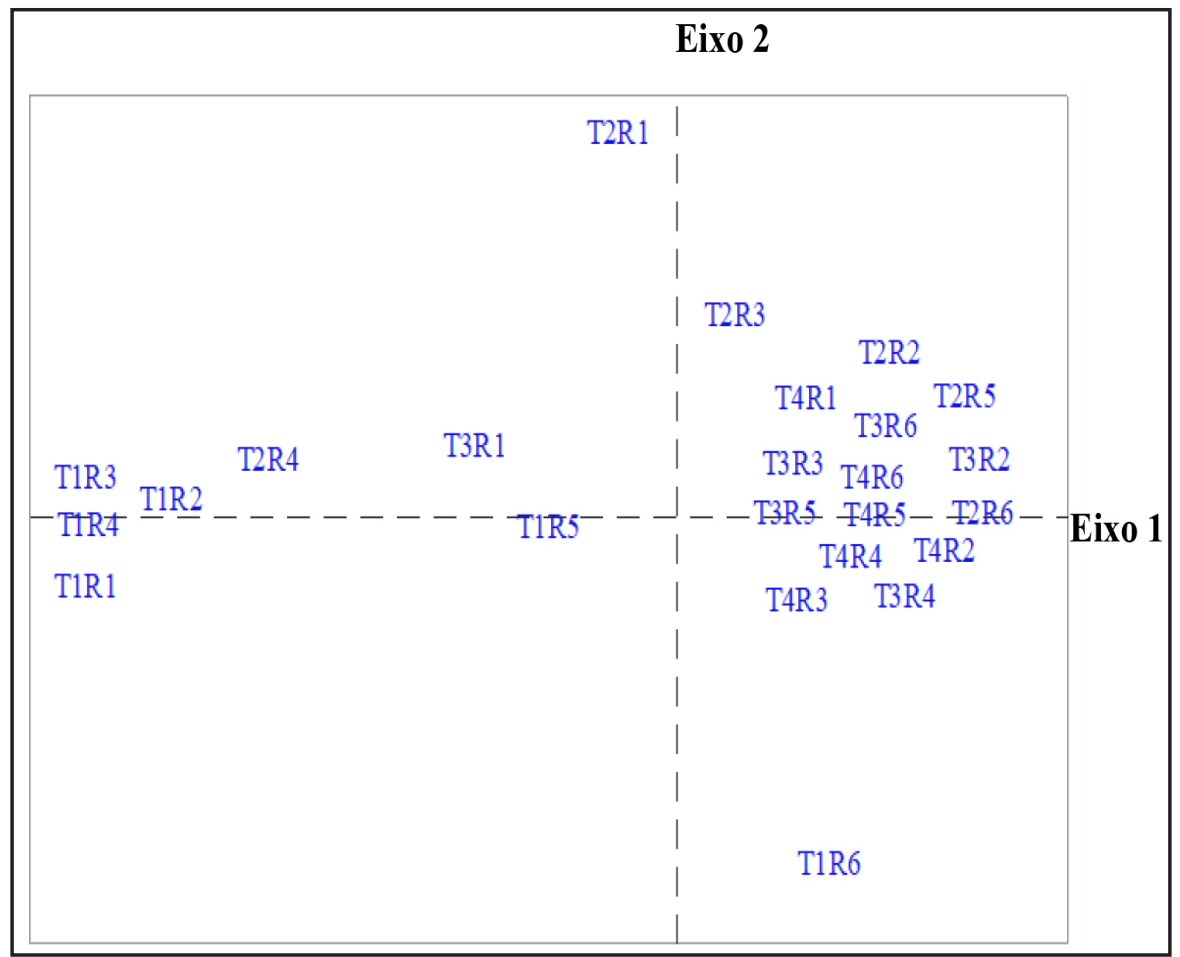

Fonte: Autores (2019)

Tabela 3 - ANOVA associada ao Modelo Linear Generalizado

Table 3 - ANOVA associated to the Generalized Linear Model

\begin{tabular}{lcccccc}
\hline \multicolumn{1}{c}{ Fatores } & H' & H'N $^{\prime}$ & $\mathbf{S}$ & $\mathbf{S N}$ & $\mathbf{C U B}(\mathbf{m})$ & $\mathbf{C N}(\mathbf{m})$ \\
\hline Herbicida & $0,007^{*}$ & $0,017^{*}$ & 0,053 & $0,047^{*}$ & 0,050 & 0,138 \\
Escarificação & $0,004^{*}$ & $0,000^{*}$ & $0,006^{*}$ & $0,012^{*}$ & 0,146 & $0,007^{*}$ \\
\hline Herbicida x Escarificação & 0,067 & 0,374 & 0,053 & 0,089 & 0,275 & 0,695 \\
\hline
\end{tabular}

Fonte: Autores (2019)

Em que: $\mathrm{H}^{\prime}$ = diversidade de Shannon geral. H’N = diversidade de Shannon de espécies nativas ao Cerrado. $\mathrm{S}=$ riqueza geral. $\mathrm{SN}$ = riqueza de espécies nativas ao Cerrado. $\mathrm{CUB}=$ cobertura absoluta de Urochloa brizantha. $\mathrm{CN}=$ cobertura absoluta de espécies nativas ao Cerrado. *Valores de probabilidade inferiores a 0,05 foram considerados significativos $(\alpha=95 \%)$. 
Figura 4 - Médias dos parâmetros fitossociológicos estimados nas parcelas testemunhas $\left(T_{1}\right)$ e nas parcelas que receberam os tratamentos $\left(T_{2}, T_{3}\right.$ e $\left.T_{4}\right)$. Médias seguidas pela mesma letra não diferem significativamente pelo teste de Tukey $(\alpha=95 \%)$. H' = diversidade de Shannon geral. H'N = diversidade de Shannon de espécies nativas ao Cerrado. $\mathrm{S}=$ riqueza geral de espécies. $\mathrm{SN}$ = riqueza de espécies nativas ao Cerrado. $\mathrm{CUB}=$ cobertura absoluta de Urochloa brizantha. $\mathrm{CN}$ = cobertura absoluta de espécies nativas ao Cerrado

Figure 4 - Means of phytosociological parameters estimated in the control plots (T1) and in the plots that received the treatments (T2, T3 and T4). Means followed by the same letter do not differ significantly by the Tukey test $(\alpha=95 \%)$. H' = diversity of Shannon general. H'N = Shannon diversity of native species to the Cerrado. $S=$ general species richness. $S N=$ richness of native species to the Cerrado. $\mathrm{CUB}=$ absolute coverage of Urochloa brizantha. $\mathrm{CN}=$ absolute coverage of native species to the Cerrado

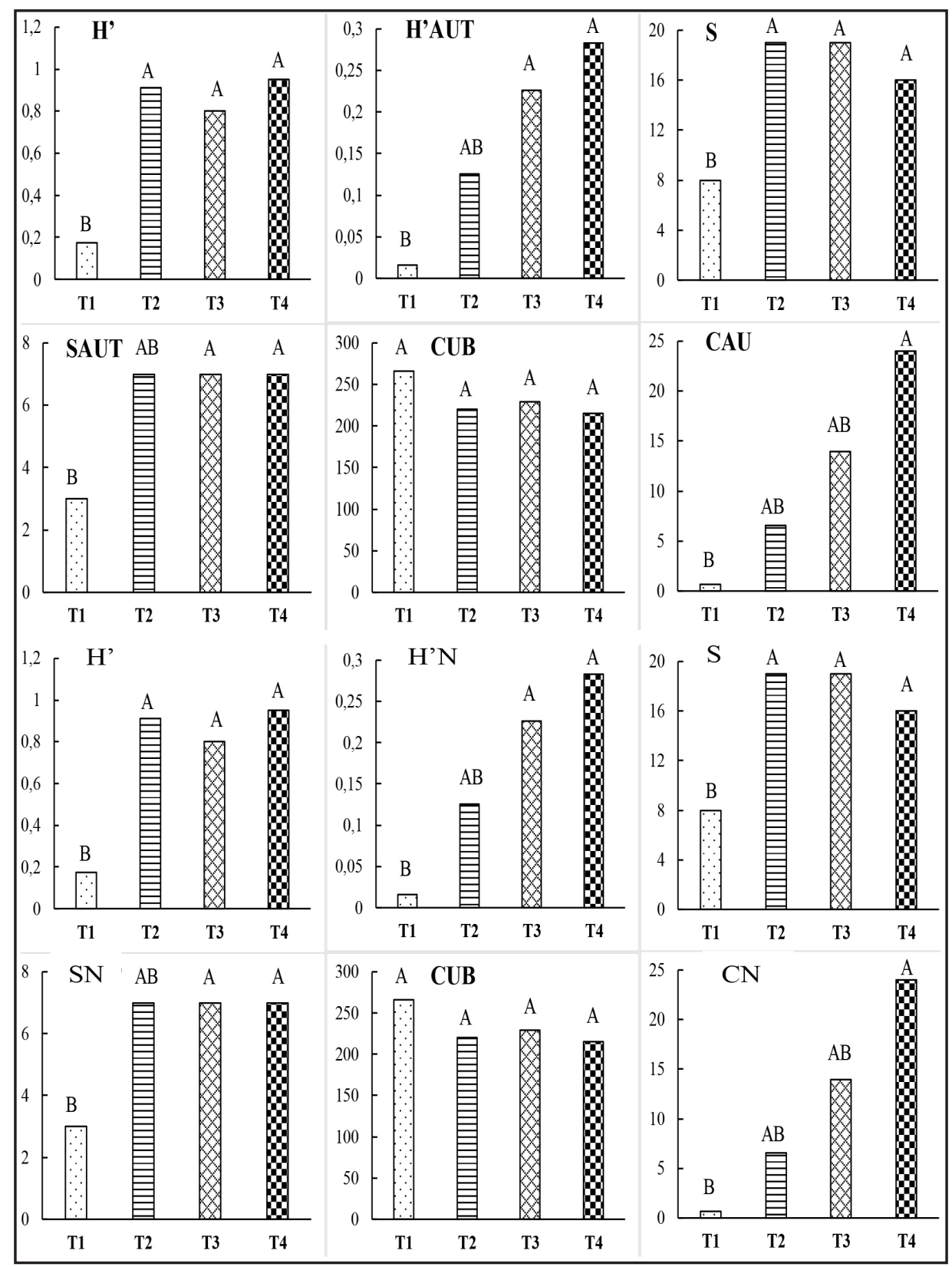

Fonte: Autores (2019) 
Comunidades vegetais invadidas por espécies exóticas, mas que apresentam vegetação nativa residual, podem ser parcialmente restauradas por meio do controle anual das invasoras (DAVIES; SHELEY, 2011; MANTOANI; DIAS; TOREZAN, 2016). A queima prévia associada à aplicação de herbicida "Imazapic", por exemplo, forneceu o melhor resultado para controlar Taeniatherum caput-medusae (gramínea anual alóctone), aumentar a diversidade de plantas e facilitar resposta positiva de grupos funcionais de espécies nativas (DAVIES; SHELEY, 2011). A remoção de plantas de espécies invasoras por capina manual ou herbicida pós-emergente resultou em maior biomassa de plantas, riqueza de espécies e regeneração de árvores em uma floresta estadunidense de clima temperado (FLORY; CLAY, 2009).

A aplicação de herbicida (Glifosato) aos 30 e aos 60 dias após a implantação das parcelas experimentais deste trabalho mostrou-se ineficaz para reduzir a cobertura absoluta da Urochloa brizantha (CUB), parcialmente eficaz para aumentar a diversidade de Shannon de espécies nativas $\left(\mathrm{H}^{\prime} \mathrm{N}\right)$, a cobertura absoluta de espécies nativas $(\mathrm{CN})$ e a riqueza de espécies nativas (SN) e eficaz para elevar significativamente os valores de diversidade de Shannon das comunidades vegetais $\left(\mathrm{H}^{\prime}\right)$ e a riqueza geral de espécies (S) (Figura 4). O controle químico de Urochloa brizantha facilitou o recrutamento de espécies na área de estudo, mas as alterações dos parâmetros calculados exclusivamente para espécies nativas $\left(\mathrm{H}^{\prime} \mathrm{N}, \mathrm{CN}, \mathrm{SN}\right)$ não foram significativas em relação às parcelas testemunhas $\left(\mathrm{T}_{1}\right)$ em até 365 dias de avaliação. A única exceção refere-se ao aumento significativo da cobertura absoluta de espécies nativas $(\mathrm{CN})$ nas parcelas em que a aplicação de herbicida foi associada à descompactação da superfície revegetada $\left(\mathrm{T}_{4}\right)$ (Figura 4).

A compactação representa um filtro abiótico que impede ou dificulta o estabelecimento de plantas em substratos expostos pela mineração (BENTO, 2009). Substratos de áreas mineradas apresentam elevada densidade, baixa porosidade e capacidade de armazenar e disponibilizar água para as plantas, baixa concentração de matéria orgânica e de nutrientes, entre outras características desfavoráveis aos vegetais (CORRÊA; BENTO, 2010). Valores de densidade aparente entre 1,2 e $1,4 \mathrm{Mg} \mathrm{m}^{-3}$ são comuns em substratos minerados na região do Cerrado e esses valores refletem condição edáfica inapropriada para a regeneração natural da vegetação em jazidas, retardando o processo de sucessão ecológica (BENTO, 2009). Nesse sentido, a recuperação de jazidas mineradas por meio da revegetação requer práticas de manejo de solo, tais como a recomposição da paisagem e a escarificação de superfícies compactadas. Essas práticas criam ambiente estável que favorece a sucessão ecológica (CORREA et al., 2018) e reduzem a resistência do substrato à penetração de raízes (CORRÊA; BENTO, 2010). A combinação de práticas de conservação de solo com a incorporação de matéria orgânica a substratos minerados influencia positivamente o desenvolvimento de cobertura vegetal e o incremento da diversidade de plantas que colonizam jazidas mineradas (BORGES et al., 2009; FRAGA, 2016; CORREAA et al., 2018).

Os ganhos ecológicos e ambientais de se recuperar uma área minerada iniciam-se com estabelecimento de cobertura vegetal no local degradado (CORREA et al., 2018). Identificar espécies nativas capazes de se desenvolverem em jazidas mineradas é fundamental para o sucesso da restauração ecológica em jazidas (SILVA et al., 2013). Contudo, o processo de regeneração natural em jazidas abandonadas à sucessão é extremamente lento (CORRÊA et al., 2018), uma vez que há fortes limitações na propagação por sementes de espécies do Cerrado (SALAZAR et al., 2012). Mesmo em locais não minerados, tais como pastagens abandonadas em diferentes estágios sucessionais, métodos passivos de restauração não têm sido capazes de recompor a estrutura e a composição florística de fitofisionomais de Cerrado (CAVA et al., 2018).

Se o objetivo da restauração for recuperar a diversidade da camada rasteira do Cerrado e manter a estrutura original da vegetação, com árvores esparsas sobre um estrato graminoide contínuo, intervenções são necessárias (BUISSON et al., 2018). Elas devem incluir o controle de espécies vegetais invasoras e a reintrodução de espécies de diferentes formas e portes de crescimento outrora existentes (SILVA et al., 2013). Todavia, não é possível apontar uma única técnica que seja recomendável para todas as situações em que se deseja restaurar fitofisionomias de Cerrado (CAVA et al., 2016). 
Neste estudo, os tratamentos aplicados para reduzir a ação dos filtros competição e compactação do substrato resultaram na redução da dominância de Urochloa brizantha e no recrutamento de 29 espécies de plantas, embora 66\% delas sejam exóticas ao Cerrado, ruderais ou invasoras. Esse recrutamento aumentou a riqueza e a diversidade de espécies de plantas nas parcelas experimentais e ganhos de diversidade são fundamentais para restaurar funções e manter ecossistemas em funcionamento (HULVEY; AIGNER, 2014).

\section{Conclusões}

A redução temporária da dominância de Urochloa brizantha, por meio da aplicação de herbicida e da escarificação da superfície compactada da jazida revegetada, permitiu que 29 espécies vegetais recrutassem no local. A recuperação da cobertura vegetal formada por Urochloa brizantha não resultou na exclusão das espécies que, espontaneamente, se estabeleceram nas parcelas experimentais. A atenuação da ação dos filtros ecológicos competição e compactação da superfície acarretaram, indistintamente, ganhos de riqueza e diversidade de espécies. Com base nos parâmetros analisados (Figura 4), os tratamentos aplicados não excluíram cobertura de Urochloa brizantha, mas melhoraram a condição ecológica das comunidades vegetais presentes nas parcelas.

\section{Agradecimentos}

Os autores agradecem ao Conselho Nacional de Desenvolvimento Científico e Tecnológico-CNPq (EditalUniversal14/2014) e à Fundação de Apoio à Pesquisa do Distrito Federal FAP/DF (Edital 6/2017) pelo suporte financeiro, ao Sr. Jorge Roberto Silveira por permitir a instalação das parcelas experimentais em sua propriedade, ao Herbário da Universidade de Brasília(UnB)e aos técnicos, professores e alunos que contribuíram para o desenvolvimento deste trabalho.

\section{Referências}

BENTO, M. A. B. Avaliação da qualidade dos substratos minerados em cinco cascalheiras revegetadas no Distrito Federal. 2009. Dissertação (Mestrado em Ciências Florestais) Universidade de Brasília, Brasília, 2009.

BORGES, T. A. et al. Uso de biossólido na recuperação de área degradada no Distrito Federal. Revista AIDIS de Ingeniería y Ciencias Ambientales, [s. l.], v. 2, n. 1, p. 65-75, 2009.

BUISSON, E. et al. Resilience and restoration of tropical and subtropical grasslands, savannas, and grassy woodlands. Biological Reviews, [s. l.], v. 94, n. 2, p. 1-19, 2018.

CARAMASCHI, G. M. C. L. et al. The superior re-sprouting performance of exotic grass species under different environmental conditions: the study case of Paspalum atratum (Swallen) and Urochloa brizantha (Hochst. ex A. Rich. - Stapf.). Theoretical and Experimental Plant Physiology, [s. l.], v. 28, n. 2, p. 273- 285, 2016. doi 10.1007/s40626-016-0058-6

CASTILLIONI, K. P. Avaliação de diferentes técnicas de manejo para controle de gramíneas invasoras em unidades de conservação de Cerrado. Dissertação (Mestrado em Ciências Biológicas) - Universidade Estadual Paulista, Rio Claro, 2015.

CAVA, M. G. B. et al. Abandoned pastures cannot spontaneously the atributes of old-growth savannas. Journal of Applied Ecology, Oxford, v. 55, p. 1164-1172, 2018.

CAVA, M. G. B. et al. Comparação de técnicas para restauração da vegetação lenhosa de Cerrado em pastagens abandonadas. Hoehnea, [s. l.], v. 43, n. 2, p. 301-315, 2016. 
CLIMATE-DATA.ORG. Dados climáticos para cidades mundiais Disponível em: https:// pt.climate-data.org/. Acesso em: 1 abr. 2018.

COMPANHIA DE PLANEJAMENTO DO DISTRITO FEDERAL. Geo Serviço e Catálogo de Mapas da CODEPLAN. Brasília, [2018]. Disponível em: http://catalogo.mapa.codeplan.df.gov.br/ e http://orto foto.mapa.codeplan.df.gov.br/demo/tms. Acesso em: 1 abr. 2018.

CORRÊA, R. S. et al. Vegetation cover development resulting from different restoration approaches of exploited mines. Revista Floresta e Ambiente,[s. l.], v. 25, n. 4, p. 1-9, 2018.

CORRÊA, R. S.; BENTO, M. A. B. Qualidade do substrato minerado de uma área de empréstimo revegetada no Distrito Federal. Revista Brasileira de Ciência do Solo, [s. l.], v. 34, n. 4, p. 1435$1444,2010$.

DAVIES, K. W.; SHELEY, R. L. Promoting native vegetation and diversity in exotic annual grass infestations. Restoration Ecology,Malden, v. 19, n. 2, p. 159-165, 2011.

EMBRAPA. Sistema Brasileiro de Classificação de Solos. Brasília: EMBRAPA Informação Tecnológica, 2013. 353 p.

FELFILI, J. M. et al.Análise multivariadas: princípios e métodos em estudos de vegetação. In: FELFILI, J. M. et al. (ed.). Fitossociologia no Brasil - Métodos e estudos de caso: Volume I. Viçosa, MG: Editora Universidade Federal de Viçosa, 2011. 556 p.

FLORY, S. L.; CLAY, K. Invasive plant removal method determines native plant community responses. Journal of Applied Ecology, Oxford, v. 46, p. 434-442, 2009.

FRAGA, L. P. Efeitos da aplicação de biossólido e resíduos de poda na revegetação de área de empréstimo no Distrito Federal. 2016. Dissertação (Mestrado em Ecologia) - Universidade de Brasília, Brasília, 2016.

GOMES, M. P. et al. Alteration of plant physiology by glyphosate and its by-product aminomethylphosphonic acid: an overview. Journal of Experimental Botany, [s. l.], v. 65, n. 17, p. 4691-4703, 2014.

HALASSY, M. et al. The application of a filter-based assembly model to develop best practices for Pannonian sand grassland restoration. Journal of Applied Ecology, Oxford, v. 53, p. 765-773, 2016.

HULVEY, K. B.; AIGNER, P. A. Using filter-based community assembly models to improve restoration outcomes. Journal of Applied Ecology, Oxford, v. 51, p. 997-1005, 2014.

JARDIM BOTÂNICO DO RIO DE JANEIRO. REFLORA - Plantas do Brasil: resgate histórico e herbário virtual para o conhecimento e conservação da flora brasileira. Rio de Janeiro, [2019]. Disponível em: http://floradobrasil.jbrj.gov.br/reflora/PrincipalUC/PrincipalUC.do. Acesso em: 1 out. 2019.

LANNES, L. S. et al. Native and alien herbaceous plants in Brazilian Cerrado are (co-) limited by different nutrients. Plant Soil, [s. l.], v. 400, n. 1-2, p. 231-243, 2015.

MANTOANI, M. C.; DIAS, J.; TOREZAN, J. M. D. Roçagem e aplicação de herbicida para controle de Megathyrsus maximus: danos sobre a vegetação preexistente em um reflorestamento de 20 anos. Ciência Florestal, Santa Maria, v. 26, n. 3, p. 839-851, 2016.

MARTINS, C. R. et al. Impacto da invasão e do manejo do capim-gordura (Melinis minutiflora) sobre a riqueza e biomassa da flora nativa do Cerrado sentido restrito. Revista Brasileira de Botânica, São Paulo, v. 34, n. 1, p. 73-90, 2011.

MUNHOZ, C. B. R.; ARAÚJO, G. M. Métodos de Amostragem do Estrato Herbáceo-subarbustivo. In: FELFILI, J. M. et al. (ed.). Fitossociologia no Brasil - Métodos e estudos de caso: Volume I. Viçosa, MG: Editora Universidade Federal de Viçosa, 2011. 556 p. 
PILON, N. A. L.; BUISSON, E.; DURIGAN, G. Restoring Brazilian savanna ground layer vegetation by topsoil and hay transfer. Restoration Ecology, Malden, v. 26, n. 1, p. 73-81, 2017.

RODRIGUES, L. de. B. Efeitos ecotoxicológicos do glifosato e formulações em diferentes organismos. 2016. Dissertação (Mestrado em Ciências Farmacêuticas) - Universidade Federal de Goiás, Goiânia, 2016.

SALAZAR, A. et al. Seed limitation of woody plants in Neotropical savannas. Plant Ecology,[s. l.], v. 213, n. 2, p. 273-287, 2012.

SILVA, L. C. R. et al. Unprecedented carbon accumulation in mined soils: the synergistic effect of resource input and plant species invasion. Ecological Applications, [s. l.], v. 23, n. 6, p. 13451356, 2013.

SOLLENBERGER, D. et al. Environmental filtering mediates grassland community assembly following restoration with soil carbon additions. Restoration Ecology, Malden, v. 24, n. 5, p. 626-636, 2016.

THOMAS, P. A. Restauração ecológica em campos invadidos por Urochloa decumbens nos campos sulinos. 2017. Dissertação (Mestrado em Ecologia) - Universidade Federal do Rio Grande do Sul, Porto Alegre, 2017. 